\title{
Distinct responses of two hepatocellular carcinoma cell lines of a similar origin to immunotherapies targeting regulatory or effector $\mathbf{T}$ cells
}

\author{
YUJI NAGAYAMA $^{1}$, WATARU HASE ${ }^{1}$, YASUHIDE MOTOYOSHI $^{1,2}$, OHKI SAITOH $^{1}$, \\ RINTAROH SOGAWA ${ }^{1}$ and KAZUHIKO NAKAO ${ }^{2}$
}

\begin{abstract}
${ }^{1}$ Department of Medical Gene Technology, Atomic Bomb Disease Institute, ${ }^{2}$ Department of Medical and Dental Sciences, Graduate School of Biomedical Sciences, Nagasaki University, 1-12-4 Sakamoto, Nagasaki 852-8523, Japan
\end{abstract}

Received January 23, 2007; Accepted February 26, 2007

\begin{abstract}
Balance between effector T cells (Teff) and regulatory $\mathrm{T}$ cells (Treg) appears to be very crucial for effective anti-tumor immunotherapy. The therapeutic efficacies of enhancement of Teff and suppression of Treg were compared between two murine hepatoma cell lines of a similar origin, MH129 and MH134. Enhancement of Teff was achieved by infection of tumor cells with adenovirus expressing glucocorticoid-induced tumor necrosis factor receptor-related protein (GITR), and suppression of Treg, by depletion of $\mathrm{CD} 4{ }^{+} \mathrm{CD} 25^{+}$naturally occurring Treg by administration of anti-CD25 monoclonal antibody (PC61) or low-dose cyclophosphamide. Our data show that MH129 cells were susceptible to Treg depletion but resistant to GITR expression, and vice versa for MH134 cells. Thus, in MH129 cells, injection of PC61 prior to or after tumor cell inoculation completely or partially, respectively, eradicated tumor growth. Low-dose cyclophosphamide administered after tumor cell inoculation also delayed tumor growth. However, GITR expression either in vitro or in vivo exhibited little effect. In contrast, in MH134 cells, PC61 induced partial tumor growth delay only when injected prior to tumor cell inoculation, and low-dose cyclophosphamide showed no effect, but GITR, particularly when administered in vitro, inhibited tumor growth. An additive effect of PC61 and GITR was observed only in MH134 cells. The ratios of peripheral $\mathrm{CD}^{+} \mathrm{CD}_{25} 5^{+}$to $\mathrm{CD} 4^{+} \mathrm{T}$ cells remained unaltered during the experimental course in both tumor models. From these results we speculate that this different sensitivity may be due to a difference in relative induction levels of Teff
\end{abstract}

Correspondence to: Dr Yuji Nagayama, Department of Medical Gene Technology, Atomic Bomb Disease Institute, Nagasaki University Graduate School of Biomedical Sciences, 1-12-4 Sakamoto, Nagasaki 852-8523, Japan

E-mail: nagayama@nagasaki-u.ac.jp

Key words: effector $\mathrm{T}$ cells, $\mathrm{CD} 4{ }^{+} \mathrm{CD} 25^{+}$regulatory $\mathrm{T}$ cells, GITRL, cyclophosphamide, PC61 versus Treg, not due to different immunogenicity or different kinetics of peripheral Treg, between the two tumor models. Future studies identifying antigen(s) or epitope(s) specific for Teff and Treg in these tumor cell lines are necessary as analysis of the immune response to such antigen(s) or epitope(s) may in general help predict the relative efficacy of different immunotherapies against distinct tumors.

\section{Introduction}

It is well known that most tumor cells possess tumorassociated antigens, many of which are found as non-mutated self-components (1). These tumor antigens however generally fail to elicit a significant anti-tumor immune response. This is because, although autoreactive effector T cells (Teff) are present in the periphery of virtually all the subjects, they are kept in check by regulatory T cells (Treg) (2). Therefore, the balance between the number and/or the function of Teff and Treg appears to be highly crucial for the outcome of antitumor immunotherapy. In other words, an effective anti-tumor immune response can be obtained not only by enhancing Teff function but also by attenuating the suppressor function of Treg.

Among the different types of Treg identified so far, $\mathrm{CD} 4{ }^{+} \mathrm{CD} 25^{+}$Treg have been evaluated most extensively. It has become increasingly clear that Treg play a critical role in autoimmunity, transplantation and also in tumor immunity (3). Thus in vivo depletion of $\mathrm{CD} 4{ }^{+} \mathrm{CD} 25^{+}$Treg with antiCD25 monoclonal antibody (PC61) has been shown to eradicate some but not all tumors in animal models (4-9). Additionally, our recent study has demonstrated that lowdose cyclophosphamide $(20 \mathrm{mg} / \mathrm{kg})$ also depletes Treg selectively and induced a significant anti-tumor immune response in a murine hepatoma cell line MH129 (10).

Glucocorticoid-induced tumor necrosis factor receptor (TNFR)-related protein (GITR), a member of the TNFR superfamily, is highly expressed in Treg (11). Although GITR expression is dispensable for Treg function (12), agonistic monoclonal antibody against GITR (DTA-1) has previously been shown to abrogate the suppressor function of Treg and consequently enhance the immune response $(13,14)$. Therefore GITR was originally considered a potential target for 
suppression of Treg function. Later, however, it was discovered that GITR is also expressed in resting $\mathrm{CD} 4{ }^{+} \mathrm{CD} 25^{-}$ and $\mathrm{CD}^{+} \mathrm{T}$ cells at low levels and was up-regulated upon activation, and the signal through GITR co-stimulated both $\mathrm{CD} 4^{+} \mathrm{CD} 25^{-}$and $\mathrm{CD} 8^{+} \mathrm{T}$ cells particularly with suboptimal $\mathrm{T}$ cell receptor stimulation $(12,15)$. Importantly, the co-culture experiments with $\mathrm{CD}^{+}{ }^{+} \mathrm{CD} 25^{-}$and $\mathrm{CD} 4{ }^{+} \mathrm{CD} 25^{+} \mathrm{T}$ cells from wild-type or GITR knockout mice revealed that ligation of GITR on Teff is required to abrogate suppression by Treg (16). Thus it is currently believed that the signal through GITR mainly activates Teff rather than suppressing Treg $(11,17)$.

In this study we evaluated the relative therapeutic efficacy of enhancement of Teff function through GITR signaling by adenovirus-mediated expression of GITR ligand (GITRL) and inhibition of suppressor function of Treg by depletion with PC61 or low-dose cyclophosphamide in two murine hepatoma cell lines, MH129 and MH134, of a similar origin.

\section{Materials and methods}

Cell lines and mice used. MH129 and MH134 cells were $\mathrm{CCl}_{4}$-induced murine hepatoma cell lines from $\mathrm{C} 3 \mathrm{H} / \mathrm{He}$ strain (18), and were maintained in RPMI-1640 medium with $10 \%$ FCS and appropriate antibiotics. In vitro growth of each cell line was evaluated with the trypan blue exclusion test.

Six-week-old female $\mathrm{C} 3 \mathrm{H} / \mathrm{HeN}$ mice were purchased from Charles River (Tokyo, Japan) and kept in a specific pathogenfree facility. All experiments were conducted in accordance with the principles and procedures outlined in the Guideline for the Care and Use of Laboratory Animals in Nagasaki University.

Preparation of anti-CD25 monoclonal antibody. Anti-CD25 monoclonal antibody was purified from ascites of nude mice intraperitoneally injected with hybridoma PC61 using HiTrap $^{\text {TM }}$ protein G HP column (Amersham, Piscataway, NJ, USA). PC61 hybridoma was from Dr K. Yui, Nagasaki University, Nagasaki, Japan.

Construction of adenovirus expressing mouse GITRL. The plasmid containing cDNA for mouse GITRL (pTG16491) was kindly provided by Dr B. Calmels, Transgene, Strasburg, France (19). GITRL cDNA was excised by digestion with NheI and SalI, and ligated into NheI- and SalI-digested bicistronic vector pIRES2-AcGFP1 (Takara, Tokyo, Japan). The DNA fragment containing the GITRL cDNA, IRES and AcGFP was then released by NheI- and NotI-digestion and ligated into NheI- and NotI-digested adenovirus shuttle vector pHMCMV6 (20). The resulting plasmid pHMGITRL-IRES-GFP was cut with PI-SceI and I-CeuI, and ligated into pAdHM15 (20). pAdHM-GITRL-IRES-GFP was linearized with $P a c \mathrm{I}$, and transfected into 293 human embryonic kidney cells to yield Ad-GITRL-IRES-GFP. The adenovirus obtained was amplified in 293 cells and purified using two rounds of $\mathrm{CsCl}$ gradient centrifugation as previously described (21). Determination of plaque-forming unit (pfu) was also performed as previously described (21).

Expression of GITRL and GFP was confirmed by FACScan flow cytometry and Cell Quest software program
(BD Biosciences, Mountain View, CA, USA) after staining the MH129 cells infected with Ad-GITRL-IRES-GFP at an MOI of 100 with PE-conjugated anti-GITRL antibody (e-Bioscience, San Diego, CA, USA).

Infectivity of MH129 and MH134 cells to adenovirus infection. The cells were infected with Ad-EGFP (a kind gift from Dr H. Yamasaki, Nagasaki University) at MOIs of 30, 100 and 300. On the next day, expression of EGFP was determined by flow cytometry as described above.

Expression of GITR on MH129 and MH134 cells. Expression of GITR on the cells was analyzed by staining the cells with PE-anti-GITR antibody (e-Bioscience) as described above.

In vivo experiments. The cells $\left(5 \times 10^{5}\right.$ cells/mouse $)$ were subcutaneously injected into the flanks of mice. Tumor sizes were determined from caliper measurement using the standard formula (length $\mathrm{x}$ width $^{2} / 2$ ) and expressed as the mean $\pm \mathrm{SE}$.

In the first series of experiments with cyclophosphamide and PC61, groups of mice were treated with either an intraperitoneal injection of cyclophosphamide (20 or $200 \mathrm{mg}$ / $\mathrm{kg}$ ) (Sigma-Aldrich, St. Louis, MO, USA) or PC61 (500 $\mu \mathrm{g} /$ mouse) four days before or after tumor cell inoculation. In vivo depletion of $\mathrm{CD}^{2} 5^{+}$cells by PC61 was confirmed by flow cytometric analysis as described above after staining splenocytes four days after an intraperitoneal injection of antibody with FITC-conjugated-anti-CD4 and PE-anti-CD25 antibodies that recognize a different epitope of CD25 (7D4) (e-Bioscience) (10).

In the second series of experiments with GITRL, groups of mice were injected with the cells infected with AdGITRL-IRES-GFP or Ad-EGFP at an MOI of 100 for $24 \mathrm{~h}$. Alternatively, other groups of mice were first injected with uninfected tumor cells, and when tumor sizes reached $\sim 5 \mathrm{~mm}$ in diameter, $5 \times 10^{8} \mathrm{pfu}$ Ad-GITRL-IRES-GFP or AdEGFP in $50 \mu 1$ PBS was injected intratumorally.

Finally, the combined effect of PC61 and Ad-GITRLIRES-GFP was also studied. Groups of mice were injected on day 0 with the cells infected with Ad-GITRL-IRES-GFP at an MOI of 100 for $24 \mathrm{~h}$, and were injected with PC61 on day +4 (MH129) or day -4 (MH134).

\section{Results}

As evident from our recent report (10) and Figs. 1 and 2 in this study, MH129 cells are susceptible to depletion of CD25+ cells. Thus, injection of PC61 prevented tumor formation completely or partially when administered four days before or after tumor cell inoculation, respectively (Fig. 1A). CD4 ${ }^{+} \mathrm{CD} 25^{+}$ $\mathrm{T}$ cell depletion by low-dose cyclophosphamide $(20 \mathrm{mg} / \mathrm{kg})$ also significantly suppressed tumor growth (Fig. 2A). In contrast, MH134 cells were resistant to depletion of CD25+ cells. As shown in Fig. 1B, injection of PC61 prior to tumor cell inoculation only partially delayed tumor growth, and injection following tumor cell inoculation contributed no effect. Low-dose cyclophosphamide also showed no significant therapeutic benefit (Fig. 2B). As we have recently demonstrated (10) and illustrated in Fig. 2, high-dose 

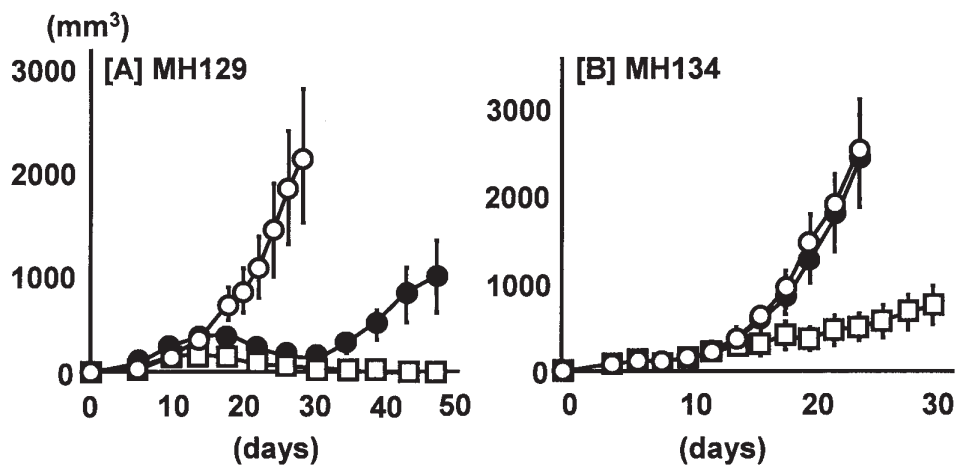

Figure 1. Anti-tumor effect of PC61 on MH129 and MH134 tumor cells in C3H/HeN mice. The mice were inoculated with 5x105 MH129 (A) or MH134 (B) cells on day 0 . Groups of mice were untreated $(\circ)$ or treated with $500 \mu \mathrm{g}$ PC61 on day $-4(\square)$ or $+4(\bullet)$. The data are the means \pm SE $(\mathrm{n}=6)$. The same experiments were repeated at least twice with similar results.
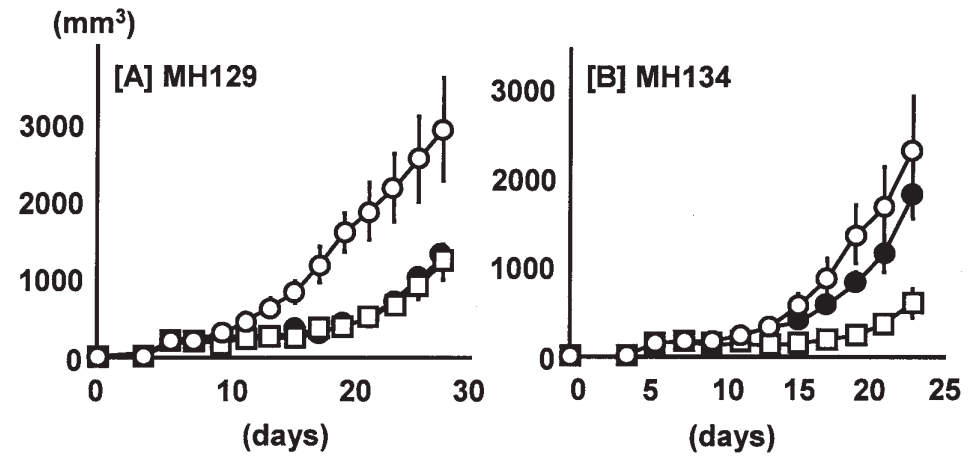

Figure 2. The anti-tumor effect of low (20 mg/kg)- and high (200 mg/kg)-dose cyclophosphamide on MH129 and MH134 tumor cells in C3H/HeN mice. The mice were inoculated with 5x105 MH129 (A) or MH134 (B) cells on day 0. Groups of mice were untreated $(\circ)$ or treated with high-dose ( $\square$ ) or low-dose $(\bullet)$ cyclophosphamide on day +4 . The data are the means \pm SE $(n=6)$. The same experiments were repeated at least twice with similar results .
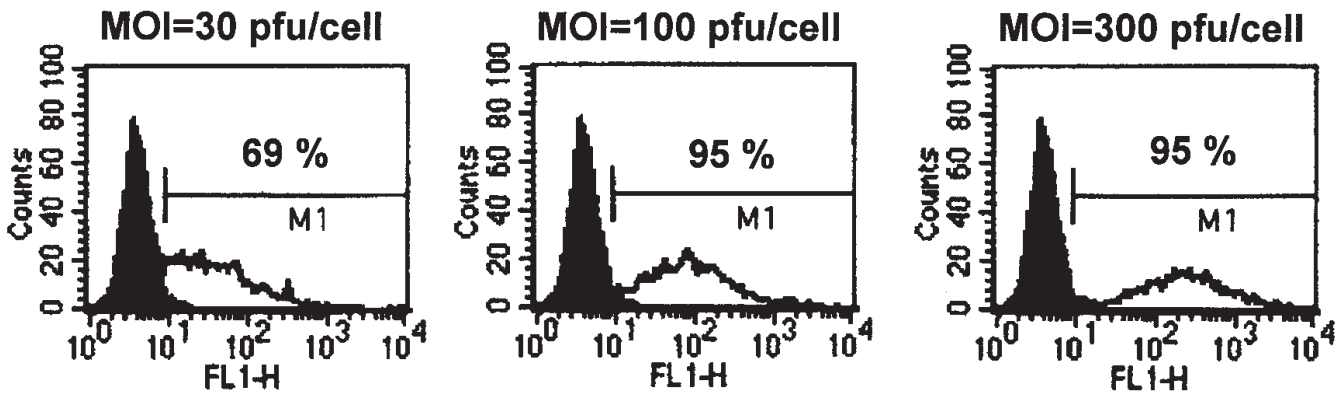

Figure 3. Flow cytometric analysis of MH129 cells infected with Ad-GITRL-IRES-GFP. The cells were untreated or infected with Ad-GITRL-IRES-GFP at an MOI of $100 \mathrm{pfu} / \mathrm{cell}$. One day later, expression of GITRL and GFP was examined as described in Materials and methods.

cyclophosphamide was equally effective in both cell lines through its direct cytotoxic effect.

Since this difference, despite the similar origin of the two cell lines, may be attributed to different kinetics of peripheral $\mathrm{CD} 4^{+} \mathrm{CD} 25^{+} \mathrm{T}$ cells in the two tumor models, the percentages of $\mathrm{T}$ cell subpopulations in splenocytes were studied. The ratios of $\mathrm{CD} 4{ }^{+} \mathrm{CD} 25^{+}$to $\mathrm{CD} 4^{+} \mathrm{T}$ cells in the spleen remained unchanged during the experimental course $(8.7 \pm 3.3 \%$ in the control; $9.2 \pm 1.2 \%$ in MH129-bearing mice at day 7 , $6.2 \pm 2.5 \%$ in $\mathrm{MH} 129$-bearing mice at day $21 ; 7.2 \pm 1.9 \%$ in MH134-bearing mice at day 7; and $8.44 \pm 0.72 \%$ in MH134bearing mice at day 21 ; mean $\pm \mathrm{SD}$ ).
The relative therapeutic effect of GITRL enhancement of Teff function in the two cell lines was next compared. To do this, recombinant, bicistronic adenovirus expressing GITRL and GFP was constructed. Expression of both molecules was confirmed by flow cytometry (Fig. 3). Ninety-five percent infectivity was observed at an MOI of 100 (Fig. 4). In vitro cell growth rates of both cells were not affected by infection with Ad-GITRL-IRES-GFP, and neither cells expressed GITR (data not shown). The in vivo effect of GITRL expression was evaluated by two different methods; one was by inoculation of adenoviral infected cells, and the other by injection of adenovirus into established tumors. 


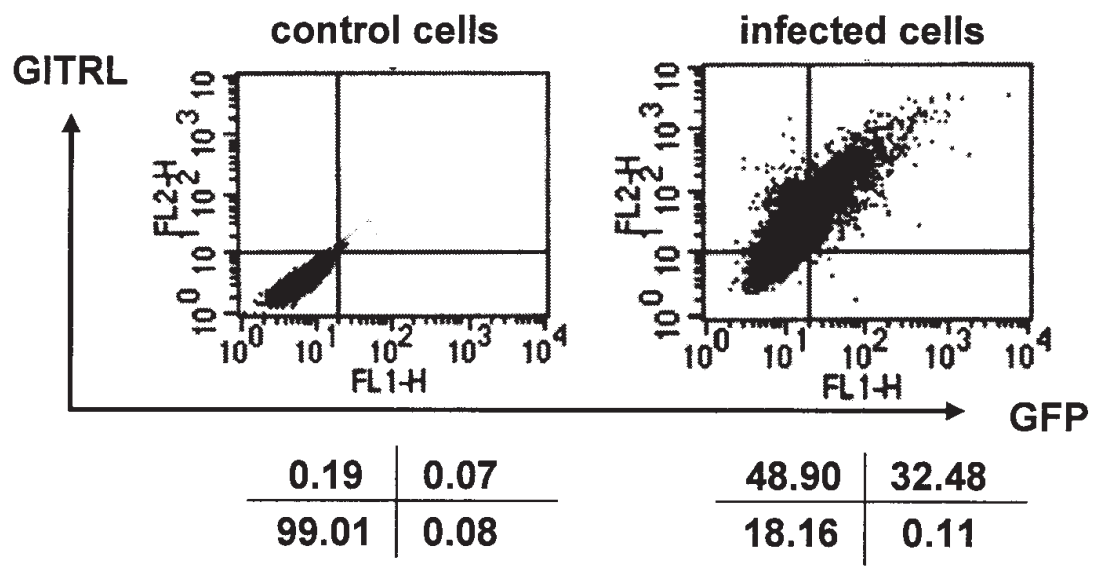

Figure 4. Flow cytometric analysis of adenovirus infectivity in MH129 cells. The cells were infected with Ad-EGFP at MOIs of 30, 100 and 300 pfu/cell. One day later, expression of EGFP was examined as described in Materials and methods.
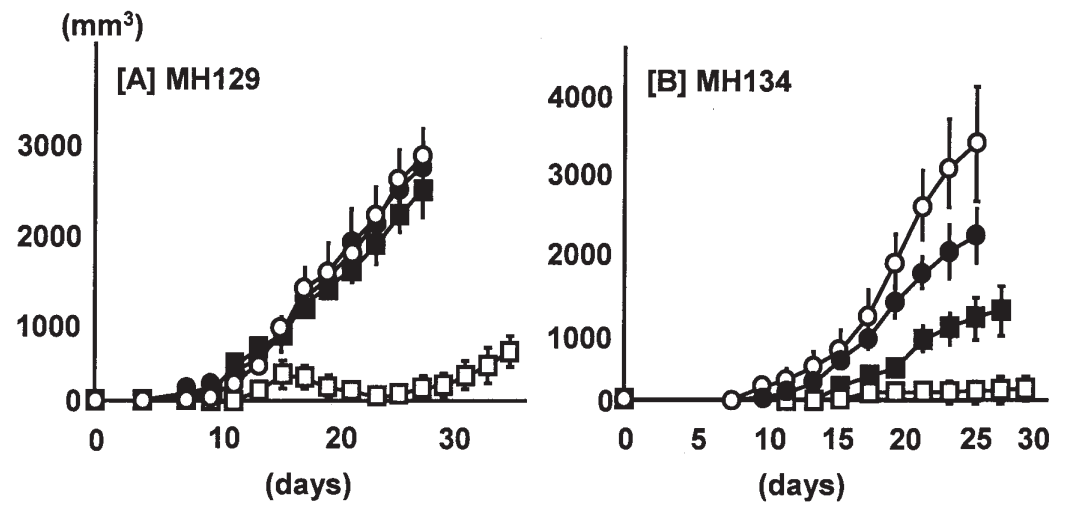

Figure 5. The anti-tumor effect of Ad-GITRL-IRES-GFP on MH129 and MH134 tumor cells in $\mathrm{C} 3 \mathrm{H} / \mathrm{HeN}$ mice. The mice were inoculated with $5 \times 10^{5}$ MH129 (A) or MH134 (B) cells infected for $24 \mathrm{~h}$ with Ad-EGFP ( $($ ) or Ad-GITRL-IRES-GFP ( $\bullet$ ) on day 0. Alternatively the mice were inoculated with $5 \times 10^{5}$ uninfected cells, and $5 \times 10^{8}$ pfu Ad-EGFP (data not shown) or Ad-GITRL-IRES-GFP $(\bullet$ ) were injected intratumorally when tumors became $\sim 5 \mathrm{~mm}$ in diameter. Furthermore, the mice were also inoculated with Ad-GITRL-IRES-GFP-infected cells and treated with PC61 four days after or before tumor cell inoculation in the MH129 and MH134 cells, respectively $(\square)$. The data are the means \pm SE (n=6-12). The same experiments were repeated at least twice with similar results.

In vivo growth of MH129 tumors was not affected by GITRL expression for either method (Fig. 5A). However, MH134 tumor growth was blunted by GITRL, particularly when the cells were infected with Ad-GITRL-IRES-GFP before inoculation (Fig. 5B). Thus, the relative responses of two hepatoma cell lines to Treg depletion and Teff enhancement are quite different; MH129 cells are susceptible to the former but resistant to the latter, and vice versa for MH134 cells.

Finally, the combined effect of PC61 and GITRL was also studied. The tumor cells were infected with Ad-GITRLIRES-GFP prior to tumor cell inoculation, and PC61 was injected four days after or before tumor cell inoculation in MH129 and MH134, respectively. The additive effect of PC61 and GITRL was observed only in MH134 cells ( $<<0.05$, Figs. 1 and 5) in the combination study.

\section{Discussion}

Effective therapeutic outcome of anti-tumor immunotherapy may be obtained by attenuating Treg suppressor function and/or enhancing Teff, for example, by depletion of Treg with PC61 or low-dose cyclophosphamide and stimulation of
Teff by GITR signal or DTA-1, respectively. The therapeutic efficacies of PC61 and GITRL or DTA-1, alone or in combination with other antigen-specific immunotherapies, have recently been documented in animal models, showing that the effects of PC61 and DTA-1 vary in different types of tumor cells. For example, Meth A fibrosarcoma cells are sensitive but B16 melanoma cells are resistant to both antibodies $(4,5,17,22,23)$. Thus it is generally accepted that Treg depletion and Teff enhancement can increase antitumor immunity against highly immunogenic tumors but has little or no effect against poorly to non-immunogenic tumors (17). However our present study clearly shows that the relative responsiveness to GITRL and PC61 is different even in two murine hepatoma cells of a similar origin.

The exact mechanism(s) of this difference are at present unclear, but may not be explained by a difference in immunogenicity of these cells, because each tumor cell line responded to at least one therapeutic approach. Also a different kinetics of peripheral Treg between these tumor models is also unlikely, because there was no difference in peripheral Treg number between these models. Instead, a difference in relative induction levels of Teff versus Treg by each cell line 
may explicate our results. From our results we suggest the following scenario. It is clear that the balance between Teff and Treg tipped toward Treg in these two tumor models, because both tumors grew well in syngeneic immunocompetent mice. However, MH129 tumor cells substantially activated both Teff and Treg (but Treg $>$ Teff). The depletion of Treg could reverse this balance, thereby leading to tumor shrinkage; however, Teff were already activated fully and GITRL was therefore ineffective. In contrast, the activation of Treg was considerably high, but that of Teff trifling in MH134 cells. The depletion of Treg by PC61 could therefore not enhance tumor immunity because of negligible Teff activity; while activated Teff by GITRL overweighed Treg and enhanced anti-tumor immunity. In these cases, activation of Treg does not necessarily mean proliferation of Treg, because the number of $\mathrm{CD} 4^{+} \mathrm{CD} 25^{+}$Treg was unaltered after tumor cell inoculation in our study. An additive effect of PC61 and GITR in MH134 cells, not in MH129, fits our hypothesis.

Notably, a recent study has shown that tumor antigen(s) prime both Teff and Treg even in the same regional lymph nodes (24), and moreover another study has also shown that certain antigen(s) selectively induce Treg (25).

To confirm our hypothesis, future studies identifying tumor antigen(s) or epitope(s) specific for Teff and Treg in these tumor cells will be required. Analysis of immune response to such antigen(s) or epitope(s) may help predict the relative efficacy of different immunotherapies against distinct tumors.

\section{References}

1. Houghton AN and Guevara-Patino JA: Immune recognition of self in immunity against cancer. J Clin Invest 114: 468-471, 2004.

2. Danke NA, Koelle DM, Yee C, Beheray S and Kwok WW: Autoreactive T cells in healthy individuals. J Immunol 172: 5967-5972, 2004

3. Sakaguchi S: Naturally rising Foxp3-expressing $\mathrm{CD} 4^{+} \mathrm{CD} 25^{+}$ regulatory $\mathrm{T}$ cells in immunological tolerance to self and nonself. Nat Immunol 6: 345-352, 2005.

4. Shimizu J, Yamasaki S and Sakaguchi S: Induction of tumor immunity by removing $\mathrm{CD} 25^{+} \mathrm{CD} 4^{+} \mathrm{T}$ cells: a common basis between tumor immunity and autoimmunity. J Immunol 163: 5211-5218, 1999 .

5. Onizuka S, Tawara I, Shimizu J, Sakaguchi S, Fujita T and Nakayama E: Tumor rejection by in vivo administration of antiCD25 (interleukin-2 receptor $\alpha$ ) monoclonal antibody. Cancer Res 59: 3128-3133, 1999.

6. Tanaka H, Tanaka J, Kjaergaard J and Shu S: Depletion of $\mathrm{CD} 4{ }^{+} \mathrm{CD} 25^{+}$regulatory cells augments the generation of specific immune $\mathrm{T}$ cells in tumor-draining lymph nodes. $\mathrm{J}$ Immunother 25: 207-217, 2002.

7. Jones E, Dahm-Vicker M, Simon AK, Green A, Powrie F, Cerundolo V and Gallimore A: Depletion of $\mathrm{CD} 25^{+}$regulatory cells results in suppression of melanoma growth and induction of autoreactivity in mice. Cancer Immun 2: 1, 2002.

8. Golgher D, Jones E, Powrie F, Elliot T and Gallimore A: Depletion of $\mathrm{CD} 25^{+}$regulatory cells uncovers immune responses to shared murine tumor rejection. Eur J Immunol 32: 3267-3275, 2002.

9. Sutmuller RP, van Duivenvoorde LM, van Elsas A, Schumacher TN, Wildenberg ME, Allison JP, Toes RE, Offringa R and Melief CJ: Synergism of cytotoxic T lymphocytes-associated antigen 4 blockade and depletion of $\mathrm{CD} 25^{+}$regulatory T cells in antitumor therapy reveals alternative pathways for suppression of autoreactive cytotoxic T lymphocyte responses. J Exp Med 194: $823-832,2001$
10. Motoyoshi Y, Kaminoda K, Saitoh O, Hamasaki K, Nakao K, Ishii N, Nagayama Y and Eguchi K: Different mechanisms for anti-tumor effects of low- and high-dose cyclophosphamide. Oncol Rep 16: 141-146, 2006.

11. Shevach EM and Stephens GL: The GITR-GITRL interaction: co-stimulation or contrasuppression of regulatory activity? Nat Rev Immunol 6: 613-618, 2006.

12. Ronchetti S, Zollo O, Bruscoli S, Agostini M, Bianchiri R, Nocentini G, Ayroldi E and Riccadi C: GITR, a member of the TNF receptor superfamily is costimulatory to mouse T lymphocyte subpopulations. Eur J Immunol 34: 613-622, 2004.

13. Shimizu J, Yamasaki S, Takahashi T, Ishida Y and Sakaguchi S: Stimulation of $\mathrm{CD} 25^{+} \mathrm{CD} 4^{+}$regulatory $\mathrm{T}$ cells through GITR breaks immunological self-tolerance. Nat Immunol 3: 135-142, 2002.

14. McHugh R, Whitters MJ, Piccirillo CA, Young DA, Shevach EM, Collins $\mathrm{M}$ and Byrne $\mathrm{MC}$ : $\mathrm{CD} 4{ }^{+} \mathrm{CD} 25^{+}$immunoregulatory $\mathrm{T}$ cells: gene expression analysis reveals a functional role for the glucocorticoid-induced TNF receptor. Immunity 16: 311-323, 2002.

15. Kanamaru F, Youngnak P, Hashiguchi M, Nishioka T, Takahashi T, Sakaguchi S, Ishikawa I and Azuma M: Costimulation via glucocorticoid-induced TNF receptor in both conventional and $\mathrm{CD} 25^{+}$regulatory $\mathrm{CD}^{+} \mathrm{T}$ cells. J Immunol 172: 7306-7314, 2004.

16. Stephens GL, McHugh RS, Whitters MJ, Young DA, Luxenberg D, Carreno BM, Collins M and Shevach EM: Engagement of glucocorticoid-induced TNFR family-related receptor on effector $\mathrm{T}$ cells by its ligand mediates resistance to suppression by CD4 ${ }^{+} \mathrm{CD} 25^{+} \mathrm{T}$ cells. J Immunol 173: 5008-5020, 2004.

17. Ramirez-Montagut T, Chow A, Hirschhorn-Cymerman D, Terwey TH, Kochman AA, Lu S, Miles RC, Sakaguchi S, Houghton AN and van den Brink MRM: Glucocorticoidinduced TNF receptor family related gene activation overcomes tolerance/ignorance to melanoma differentiation antigens and enhances antitumor immunity. J Immunol 176: 6434-6442, 2006.

18. Shima J, Yoshioka T, Kosugi A, Ogata M, Fujiwara H and Hamaoka T: Differential ability of tumor-unique and crossreactive antigen(s) on two murine hepatoma cell lines to induce Lyt-1 ${ }^{+}-\mathrm{T}$ cells responsible for in vivo protective immunity. BIKEN J 30: 1-8, 1987.

19. Calmels B, Paul S, Futin N, Ledoux C, Stoeckel F and Acres B: Bypassing tumor-associated immune suppression with recombinant adenovirus expressing membrane bound or secreted GITR-L. Cancer Gene Ther 12: 198-205, 2005.

20. Mizuguchi H, Koizumi N, Hosono T, Utoguchi N, Watanabe Y, Kay MA and Hayakawa T: A simplified system for constructing recombinant adenoviral vectors containing heterologous peptides in the HI loop of their fiber knob. Gene Ther 8: 730-735, 2001.

21. Nagayama Y, Nishihara E, Namba H, Yokoi H, Hasegawa M, Mizuguchi H, Hayakawa T, Yamashita $S$ and Niwa $M$ : Targeting the replication of adenovirus to $p 53$-defective thyroid carcinoma with a $p 53$-regulated Cre-loxP system. Cancer Gene Ther 8: 36-44, 2001

22. Ko K, Yamasaki S, Nakayama K, Nishioka T, Hirota K, Yamaguchi T, Shimizu J, Nomura T, Chiba T and Sakaguchi S: Treatment of advanced tumors with agonistic anti-GITR mAb and its effects on tumor-infiltrating Foxp $3{ }^{+} \mathrm{CD} 25^{+} \mathrm{CD} 4^{+}$ regulatory T cells. J Exp Med 202: 885-891, 2005.

23. Cohen AD, Diab A, Perales MA, Wolchok JD, Rizzuto G, Merghoub T, Huggins D, Liu C, Turk MJ, Restifo NP, Sakaguchi S and Houghton AN: Agonist anti-GITR antibody enhances vaccine-induced $\mathrm{CD}^{+} \mathrm{T}$-cell responses and tumor immunity. Cancer Res 66: 4902-4912, 2006.

24. Hiura T, Kagamu H, Miura S, Ishida A, Tanaka H, Tanaka J, Gejyo $\mathrm{F}$ and Yoshizawa $\mathrm{H}$ : Both regulatory $\mathrm{T}$ cells and antitumor effector $\mathrm{T}$ cells are primed in the same draining lymph nodes during tumor progression. J Immunol 175: 5058-5066, 2005.

25. Nishikawa H, Kato T, Tanida K, Hiasa A, Tawara I, Ikeda H, Ikarashi Y, Wakasugi H, Kronenberg M, Nakayama T, Taniguchi M, Kuribayashi K, Old LJ and Shiku H: $\mathrm{CD}^{+} \mathrm{CD}^{+} 5^{+}$ $\mathrm{T}$ cells responding to serologically defined autoantigens suppress antitumor immune responses. Proc Natl Acad Sci USA 100: 10902-10906, 2003 